\title{
Sound Pressure Level Spectra of Automotive Side- View Mirror Models Deduced From Time-Resolved Three-Dimensional Particle Tracking Velocimetry Data With Artificial Intelligence Based Data Assimilation Method
}

Dong Kim

Pusan National University

Arman Safdari

Ton Duc Thang University

Kyung Chun Kim ( $\nabla$ kckim@pusan.ac.kr)

Pusan National University

Research Article

Keywords: particle image velocimetry, artificial intelligence, data assimilation, sound pressure level spectrum, automotive side-view mirror models

Posted Date: December 11th, 2020

DOl: https://doi.org/10.21203/rs.3.rs-122714/v1

License: (9) This work is licensed under a Creative Commons Attribution 4.0 International License. Read Full License 


\section{Abstract}

This paper proposes a data assimilation method based on artificial intelligence (Al) to obtain sound level spectrum as increasing the spatial and temporal resolution of time-resolved three-dimensional Particle Tracking Velocimetry (4D PTV) data. A 4D PTV has used to measure flow characteristics of three side mirror models adopting the Shake-The-Box (STB) algorithm with four high-speed cameras on a robotic arm for measuring industrial scale. Helium filled soap bubbles are used as tracers in the wind tunnel experiment to characterize flow structures around automobile side mirror models. Full volumetric velocity fields and evolution of vortex structures are obtained and analyzed. Instantaneous pressure fields are deduced by solving a Poisson equation based on the 4D PTV data. To increase spatial and temporal resolutions of velocity field, artificial intelligence (Al)-based data assimilation method has applied. Adaptive Neural Fuzzy Inference System (ANFIS) based machine learning algorithm works well to find hidden 3D vortical structures behind the automobile side mirror model. Using the high resolution ANFIS model, power spectrum of velocity fluctuations and sound level spectrum of pressure fluctuations are successfully obtained to assess flow and noise characteristics of three different side mirror models.

\section{Introduction}

Most drivers know that they must turn up the volume to listen to their favorite radio channels on the highway and speak louder to talk to the passenger. This is a direct result of the turbulence that induced pressure fluctuation around window. The pressure fluctuation creates vibrations of window glass plates, which generate some of the internal noise through the air inside the vehicle. There are five types of wind noise sources: turbulent boundary layers, separated and reattaching flow, cavity flow, vortex shedding, and leak (or aspiration) noise [1]. Side view mirrors can contribute to significant interior noise in the automobile cabins. The vortices induced by the mirrors produce powerful exterior noise and hydrodynamic impingement, which excite the downstream windows. The interior noise can be reduced by suppressing the turbulent flow separation on the mirrors.

To measure noise sources, wind tunnel testing is still a common method in the automotive industry [1-8]. Various turbulent structures around exterior mounted vehicle mirrors have been investigated experimentally by Rinoshika et al. [9]. In addition, Khalighi et al. [10] conducted PIV and pressure measurements behind the two outer side view mirrors of the vehicle in the wake region. Kim et al. [7] measured surface flow and wake structure of passenger car side view mirror. In order to measure velocity and pressure which are important properties for determining aerodynamic performance, point measurement methods such as a pressure transducer and a hot-wire are adopted or 2D planar PIV measurement is being conducted. In the case of pressure measurement, it cannot identify the nature of the noise mechanism as well as time and effort, as the microphone arrangement is installed to measure floating noise and to use a trial-and-error method to modify features. Far field data using microphone arrays do not provide enough information on the source mechanism, so it relies on a numerical analysis or source model to understand additional information from the source region. In addition, most PIV measurements are limited to 2D. Obtaining pressure field after performing 3D flow analysis by direct 
numerical simulation (DNS) or large eddy simulation (LES) is difficult to apply to a complex shape or a high Reynolds number due to computational cost and turbulence model. In addition, the results of the numerical analysis must be verified by experiment.

Today, pressure from PIV techniques provide an alternative to the complex devices of a wind tunnel model with pressure tap for pressure measurement [11-13]. Once velocity material derivatives are accurately calculated, the established time-resolved pressure from PIV procedure solves the incompressible Poisson equation for pressure. Due to uncorrelated random errors in consecutive PIV snapshots, recent studies have employed a Lagrangian tracking [14] approach to obtain the velocity material derivative from a series of consecutive time-resolved velocity measurements. The combination of tracer particle technology for large-scale wind tunnel experiments (helium filled soap bubbles (HFSB)) and coaxial volumetric velocimetry with the advancements in 3D particle motion analysis (Shake-theBox) has shown the potential to evaluate the velocity and pressure field in volumes of several liters [15]. An accurate determination of the velocity material derivative in turbulent flows requires full $3 \mathrm{D}$ evaluation of the velocity and acceleration field, which is currently possible by high-speed, high-resolved experiment.

Turbulent flow generally involves multidimensional physics, including space and time, which have high dimensions with rotating and transforming intermittent structures. This feature provides an opportunity for the application of artificial intelligence (Al) methods, such as machine learning to predict the modeling and analysis of turbulent flow. Neural networks are popular in a range of areas, including self-driving cars and weather forecasts. Recently, there have been interesting studies on the use of neural networks for fluid dynamics [16-25], including turbulence modeling [18, 21]. The Adaptive Neuro-Fuzzy Inference System (ANFIS) method can learn many physical models and can be very useful in chemical engineering, pharmaceuticals, and industry [26]. This method calculates the changes in linguistic concepts into mathematical or computational ones, which can alter its behavior in different environments and learn to calculate the behavior of many processes that may be uncertain. Neuro-fuzzy computing methods are an example of such techniques. These methods take advantages of machine learning capability of artificial neural networks, and fuzzy inference systems to make reasonable decisions according to the if-then rules [27].

This paper proposes an Al-based data assimilation technique combining the 4D robotic PTV measurement data and an ANFIS framework for fluid dynamic research. Learning 3D or 4D flow patterns through Al can result in excellent generalization ability of the resulting model and a decrease in error and noise because it learns statistical data based on the neural network algorithm, which can be stored in a database. The instantaneous pressure field was estimated by solving the Poisson equation using 4D PTV data. The ANFIS model was trained using the time-resolved three-dimensional PTV data measured in the wake region of the side-view mirror model for automobiles as a ground truth. To determine the threedimensional flow structure and noise characteristics of the side-view mirror model, the instantaneous velocity and pressure field were deduced from the high-resolution ANFIS model. Vortex shedding phenomena and flow-induced noise are discussed based on the power spectrum and sound pressure level spectra. 


\section{Methods}

\section{D Lagrangian robotic PTV measurements of side-view mirror models.}

In this study, the time-resolved three-dimensional velocity field of the flow passing through the side-view mirror models was measured using a robotic PTV equipped with four high-speed cameras and the ShakeThe-Box (STB) algorithm. To characterize the flow structures around three different automotive side-view mirror models, helium-filled soap bubbles were used as tracers in the wind tunnel experiment as shown in Fig. 1. The coaxial volumetric velocimeter (CVV) probe was installed on a collaborative robotic arm UR5 from Universal Robots [28]. The reader should refer to the work by our previous research for a detailed discussion of the measurement system and calibration procedures [15].

\section{Pressure field evaluation from 4D PTV data.}

Instantaneous pressure, $p$, can be calculated by solving the Poisson equation [11],

$$
\nabla^{2} p=\nabla \cdot\left(-\rho \frac{D u}{D t}+\mu \nabla^{2} \boldsymbol{u}\right)
$$

with the von Neumann boundary conditions on all volume boundaries except for the top side. At the top side, a Dirichlet boundary condition is specified from the Bernoulli equation. At the boundaries, the von Neumann condition was applied, as proposed by Ebbers and Farnebäck [12]. The application of the von Neumann boundary condition will yield the solution of the pressure field up to a finite integration constant. To eliminate the latter, the Dirichlet condition needs to be specified at a known reference location. For the present data, the pressure far upstream of the test object was matched to the expected free-stream pressure. Visualization of the vorticity distribution confirmed the irrotational flow at the top boundary of the measurement. The material derivative in Eq. (1) was evaluated using the Lagrangian technique [13].

\section{Data assimilation using Adaptive Neuro-Fuzzy Inference System (ANFIS).}

Fig. 2 shows the structure of the ANFIS model used in this study. ANFIS is a kind of artificial neural network that is based on fuzzy inference system [29]. Since it integrates both neural networks and fuzzy logic principles, it has potential to capture the benefits of both in a single framework. Its inference system corresponds to a set of fuzzy IF-THEN rules that have learning capability to approximate nonlinear functions. More details on ANFIS can be found elsewhere [26, 27, 29]. The ANFIS model was used to predict the 4D flow characteristics of the side-mirror model with two membership functions as input. Four inputs of the $x, y, z$ coordinates, and time $t$ were applied to obtain the time-resolved three velocity components, and the output was the instantaneous 3D velocity components. In this study, $70 \%$ of the experiment results were used as input to the ANFIS for training. The remaining data were used as testing data to check the prediction results. The computations were performed on a computer with an Intel ${ }^{\circledR}$ Core $^{\text {TM }}$ i5-8400 CPU @ 2.80 GHz 2.81 GHz and 16.0 GB of RAM. With 300 epochs and 2 input membership functions, the ANFIS training satisfied the convergence criterion of RMSE $<0.01$. 


\section{Results And Discussion}

The ANFIS method can predict the time-resolved three-dimensional velocity field of the side mirror model with less computational time and provide high temporal and spatial resolution. The number of raw data in the $\mathrm{x} / \mathrm{H}, \mathrm{y} / \mathrm{H}$, and $\mathrm{z} / \mathrm{H}$ mesh coordinates was $28 \times 30 \times 26$ nodes, which have 21,840 data. This coordinate had a step size of 0.05 between the nodes. For ANFIS-based data assimilation of 4D PTV, the ANFIS model predicted $\mathrm{x} / \mathrm{H}, \mathrm{y} / \mathrm{H}$, and $\mathrm{z} / \mathrm{H}$ from 0 to $1.5,-0.7$ to 0.7 , and 0 to 1.3 , respectively, with step sizes of 0.00625 . This means that the spatial resolution of the raw data will increase eight-fold. The total number of nodes is $241 \times 225 \times 209$ nodes (11,333,025 data).

Fig. 3 compares the ensemble-averaged streamwise vortex structures between the raw data and ANFIS data assimilation for three different side mirror models. The increase in spatial resolution means that the vorticity, which is a function of the gradient of velocity and space, can be well distinguished. In the case of the ANFIS model, the connection of the horseshoe vortex from the bottom of the model was very clear. On the other hand, in the case of raw data, the horseshoe vortex was broken due to a lack of spatial resolution, as shown in model 1's result. The sidewall roll-up vortex and trailing vortex pair were also recovered well by ANFIS data assimilation. When the flow was developing downstream, the vortex pair inclined towards the ground plane owing to the downwash effect. The streamwise trailing vortex pair showed a dipole distribution, counterclockwise rotation in the left-hand side vortex, and clockwise rotation in the right-hand side vortex. For model 2 , the horseshoe vortex disappeared due to the inclination of the model at the front side, but a trailing vortex formed into a dipole formation. In the case of the lower vortex pairs of the dipole trailing vortex, the raw data show that the vortex form is broken. After ANFIS data assimilation, this vortex was recovered by the high spatial resolution. For model 3 , the horseshoe vortex that occurs at the base of the model is clearer. A horseshoe vortex with small size and magnitude occurs because the base of the model has the same shape as the reference model. Therefore, data assimilation can recover this missing data by increasing the spatial resolution.

The average velocity fields cannot resolve relatively small vortex structures as well as the evolution of the vortex structures. To take more advantages of the ANFIS data assimilation, the instantaneous velocity field of the side mirror model 1 was used for data assimilation. Fig. 4 presents the instantaneous streamwise vortex structures in the wake of the side mirror model 1 . The raw data at time $t_{1}$ and $t_{2}$ are shown in Fig. 4 (a) and (b). Compared to the ensemble-averaged results (Fig. 3 (a) and (b)), the effects of data assimilation were certainly apparent. The Al-based data assimilation results (Fig. 4 (c)-(f)) showed much smaller vortex structures as a result of the four-fold increase in the spatial resolution. Moreover, the four-times higher temporal resolution of the ANFIS model revealed much more small-scale streamwise vortical structures than those from only an enhancement of the spatial resolution. Compared to the average streamwise vortex structure, the instantaneous vorticity field was not a symmetrical feature. Clusters of the vortex structure rotating clockwise or counterclockwise were inclined to the bottom with downwash flow. During the measurement period of Fig. 4 (c)-(f), the vortex structures rotating clockwise were above the vortex structures rotating counterclockwise, but at other times it could be the opposite considering that the time-averaged streamwise trailing vortex structure is symmetrical. These data 
assimilation results provide a better understanding of the small turbulence structures and allow for more in-depth analysis by recovering the missed data due to the resolution limit of the experiment.

From the ANFIS model with improved temporal and spatial resolution, it was possible to extract the instantaneous velocity and pressure fluctuations at a specific location in the flow field. After obtaining the power and noise level spectrum from the time series data, it was possible to obtain the shedding frequency of the vortex from the side mirror model and identify the noise source from the fluctuation of the flow pressure. Fig. 5 shows three locations where the velocity and pressure are extracted from the horizontal plane at $\mathrm{z} / \mathrm{H}=0.5$ for model 1. Position $1(\mathrm{P} 1)$ is in the recirculation zone $(\mathrm{x} / \mathrm{H}=0.2, \mathrm{y} / \mathrm{H}=$ -0.2 ), where backflow occurs behind the model. Position 2 (P2) is located at the wake shear layer region $(\mathrm{x} / \mathrm{H}=0.5, \mathrm{y} / \mathrm{H}=-0.3)$ with a high streamwise velocity. Position $3(\mathrm{P} 3)$ is in the area where the trailing vortex appeared near the central region $(x / H=1.0, y / H=-0.1)$ of the wake after passing the recirculation zone downstream of the model.

Fig. 6 (a) shows the extracted streamwise velocity fluctuations from the ANFIS model at three different positions on model 1 for the entire measurement time, 22 seconds. The velocity magnitudes were mostly negative at $\mathrm{P} 1$ because the location is within the recirculating zone. The velocity fluctuation at $\mathrm{P} 2$ had the highest magnitude because the location is just outside of the recirculation zone, where the separated shear layer exists. The time series of the streamwise velocity at P3 had a lower velocity magnitude than that of P2. On the other hand, the turbulent intensity of P3 was higher than that of P2. The position was located in the trailing vortex region, which is inside the wake flow. Fig. 6 (b) compares the instantaneous velocity extracted at three different points from the raw velocity data and the ANFIS model with a fourfold higher temporal resolution than the raw data. For a better comparison, only 1.5 seconds were selected. The overall change was consistent with each other, but the ANFIS model resolved more fluctuations. Every fourth ANFIS result coincided with the raw data because raw data was used as the ground truth in ANFIS learning.

The dominant characteristics of the external flow over a bluff body were vortex shedding, and flowinduced noise is closely related to this phenomenon. The shape of the side mirror model was similar to a half-cylinder and vortex shedding occurred behind the model. Fast Fourier Transform (FFT) analysis was performed with the velocity signals of Fig. 6 (a). Fig. 7 shows the power spectra of the streamwise velocity fluctuations extracted at the three points. Because the power spectrum was obtained only with the fluctuation component of the velocity, the highest power value came out from position P1, where the fluctuation was the largest, and in the order of P3 and P2. In Fig. 7, the power spectra obtained using the raw velocity data and the instantaneous velocity extracted from the ANFIS model were compared. Both coincided with each other in the low-frequency range below $3 \mathrm{~Hz}$, but there were significant differences in the high-frequency range above $10 \mathrm{~Hz}$. The power spectrum obtained using the raw data revealed a noisy spectrum with the same mean value above $10 \mathrm{~Hz}$. Because the sampling rate of raw data was $289 \mathrm{~Hz}$, the spectrum above $145 \mathrm{~Hz}$ was meaningless using the Nyquist sampling criteria, and high power at a lower frequency was derived from aliasing. On the other hand, the ANFIS model had a resolution of 867 $\mathrm{Hz}$. Therefore, it showed a very clean spectrum in the frequency range below $400 \mathrm{~Hz}$. 
The instantaneous velocity field obtained as a result of the Al-based data assimilation showed the vortex shedding frequency as a very clear peak. The power spectrum tended to decrease with the power-law as it goes into higher frequencies. Interestingly, the peak frequency of the spectrum obtained at each point was different because the structure of the dominant vortex in each position was different. The most prominent frequency peak was $50 \mathrm{~Hz}$ measured at the $\mathrm{P} 1$ position. This position is closely related to the vortex shedding that occurs on the side of the semi-cylindrical side mirror model. In the P2 position, periodic vortex shedding of approximately $10 \mathrm{~Hz}$ occurred. This location was related to the abnormality of the trailing vortex structure. In the spectrum at the P3 position, a low-frequency peak of $1.5 \mathrm{~Hz}$, which was not found in the raw data, appeared in the ANFIS model results. Because the P3 position becomes the point where the recirculating zone ends, it was assumed that it would be related to the slow meandering phenomenon of the separation bubble.

The pressure fluctuation was quantified to compare the noise of the mirror models at P1. Instantaneous pressure fields were deduced by solving a Poisson equation based on the 4D PTV data. The instantaneous pressure extracted from the raw data and the ANFIS model with a four-fold higher temporal resolution. The instantaneous pressure data were converted to sound pressure levels using the following equation and a frequency analysis:

$$
\mathrm{SPL}=20 \times \log 10\left(\frac{P}{P_{\text {ref }}}\right)
$$

$P$ is the instantaneous pressure data, and $P_{\text {ref }}$ is the reference sound pressure $\left(20 \times 10^{-6}\right.$ pa was used for sound pressure in air). Figure 8 shows a comparison of the sound pressure level for different side mirror models. The magnitude of the noise level was highest for model 1 , followed by model 2 and model 3 at the same position. The peak of model 1 was dominant at $10-100 \mathrm{~Hz}$ in the low frequency band. This region has strong air resonance, and most of the noise felt by humans is in this area. At model 1 , the peak frequencies were found at $10,20,40,80$, and $120 \mathrm{~Hz}$, which are the harmonics based on $10 \mathrm{~Hz}$. At model 2 , the peak sound frequencies were observed at 50,100 , and $200 \mathrm{~Hz}$, which are the harmonics of vortex shedding frequency, $50 \mathrm{~Hz}$. At model 3, peaks appeared at 100 and $200 \mathrm{~Hz}$, the same as model 2. Models 2 and 3 have peaks in the mid frequency band but not the low frequency bands, which affects noise.

\section{Conclusions}

An Al-based data assimilation technique was developed using 4D robotic PTV measurement for fluid dynamics. By learning 3D or 4D flow patterns through Al, generalization ability of the model was obtained, and error and noise were reduced because statistical data were learned based on a neural network algorithm. The learned data can be stored in a database. Using this database, any desired data can be acquired without further experiments or computational analyses. In addition, 4D flow measurements of side mirror models were performed to experimentally investigate the 3D flow characteristics. In the case of model 1 , the formation of a horseshoe vortex from the bottom of the model was very clearly observed. In the case of model 2 , the horseshoe vortex disappeared due to the inclination 
of the model at the front side, but a trailing vortex formed into a dipole formation. In the case of the lower vortex pairs of the dipole trailing vortexes, the raw data showed that the vortex form is broken. After the ANFIS data assimilation, the vortex was recovered by the high spatial resolution. In the case of model 3, a horseshoe vortex occurred at the base of the model and was observed more clearly. For the instantaneous result, compared with raw data, the data assimilation results showed a small vortex structure as a result of increasing the spatial resolution. These data assimilation results provide a better understanding of the small turbulence structures and allow for more in-depth analysis by recovering missed data. The instantaneous pressure fields were deduced by solving a Poisson equation based on the 4D PIV data and the ANFIS method. The magnitude of the noise level was highest for model 1, and the peak was dominant at $10-100 \mathrm{~Hz}$ in the low frequency band, where humans feel noise. Models 2 and 3 had peaks in the mid-frequency band and not the low frequency band. The ANFIS model could help with numerical and experimental methods to optimize case studies without doing experiments. This method could also enable mesh refinement with low computational time.

\section{Declarations}

\section{Acknowledgement}

This research was supported by Basic Science Research Program through the National Research Foundation of Korea (NRF) funded by the Ministry of Education (No. 2020R1A6A3A03038341) and the Korean government (MSIT) (No. 2018R1A2B2007117, No. 2020R1A5A8018822).

\section{Author Contributions}

D. K. conducted experiments, D. K. and A. S. designed Al framework. D. K. wrote the manuscript and prepared all figures. K. C. K. supervised experiments, Al frameworks and proof-reading of the manuscript. All authors reviewed the manuscript.

\section{Competing interests}

The authors declare no competing interests.

\section{References}

1. Yu, C. (2017). Automotive Wind Noise Prediction using Deterministic Aero-Vibro-Acoustics Method. In 23rd AIAA/CEAS Aeroacoustics Conference (p. 3206).

2. Yao, H. D., \& Davidson, L. (2018). Generation of interior cavity noise due to window vibration excited by turbulent flows past a generic side-view mirror. Physics of Fluids, 30(3), 036104.

3. Wang, Y., Gu, Z., Li, W., \& Lin, X. (2010). Evaluation of aerodynamic noise generation by a generic side mirror. World Academy of Science, Engineering and Technology, 61, 364-371.

4. Wang, Q., Chen, X., Zhang, Y., \& Meng, W. (2018). Unsteady Flow Control and Wind Noise Reduction of Side-View Mirror (No. 2018-01-0744). SAE Technical Paper. 
5. Wan, J., \& Ma, L. (2017). Numerical investigation and experimental test on aerodynamic noises of the bionic rear view mirror in vehicles. Journal of Vibroengineering, 19(6), 4799-4815.

6. Vanherpe, F., Baresh, D., Lafon, P., \& Bordji, M. (2011). Wavenumber-frequency analysis of the wall pressure fluctuations in the wake of a car side mirror. In 17th AIAA/CEAS Aeroacoustics Conference (32nd AIAA Aeroacoustics Conference) (p. 2936).

7. Kim, J. H., Han, Y. O., Lee, M. H., Hwang, I. H., \& Jung, U. H. (2008). Surface flow and wake structure of a rear view mirror of the passenger car. Proceedings of the Bluff Bodies Aerodynamics \& Applications (BBAA) VI, Milan, Italy, 20-24.

8. Kharazi, A., Duell, E., Kimbrell, A., \& Boh, A. (2015). Prediction of Flow-Induced Vibration of Vehicle Side-View Mirrors by CFD Simulation (No. 2015-01-1558). SAE Technical Paper.

9. Rinoshika, A., \& Watanabe, S. (2010). Orthogonal wavelet decomposition of turbulent structures behind a vehicle external mirror. Experimental thermal and fluid science, 34(8), 1389-1397.

10. Khalighi, B., \& Lee, R. (2011). PIV Velocity and Pressure Measurements of the Unsteady Flow Field behind Two Automobile Outside Rear View Mirrors.

11. Van Oudheusden, B. W. (2013). PIV-based pressure measurement. Measurement Science and Technology, 24(3), 032001.

12. Ebbers, T., \& Farnebäck, G. (2009). Improving computation of cardiovascular relative pressure fields from velocity MRI. Journal of Magnetic Resonance Imaging: An Official Journal of the International Society for Magnetic Resonance in Medicine, 30(1), 54-61.

13. Pröbsting, S., Scarano, F., Bernardini, M., \& Pirozzoli, S. (2013). On the estimation of wall pressure coherence using time-resolved tomographic PIV. Experiments in fluids, 54(7), 1567.

14. Schanz, D., Gesemann, S., \& Schröder, A. (2016). Shake-The-Box: Lagrangian particle tracking at high particle image densities. Experiments in fluids, 57(5), 70.

15. Kim, D., Kim, M., Saredi, E., Scarano, F., \& Kim, K. C. (2020). Robotic PTV study of the flow around automotive side-view mirror models. Experimental Thermal and Fluid Science, 119, 110202.

16. Brunton, S. L., Noack, B. R., \& Koumoutsakos, P. (2020). Machine learning for fluid mechanics. Annual Review of Fluid Mechanics, 52, 477-508.

17. Akdemir, B., Doğan, S., Aksoy, M. H., Canli, E., \& Özgören, M. (2015, March). Artificial frame filling using adaptive neural fuzzy inference system for particle image velocimetry dataset. In Sixth International Conference on Graphic and Image Processing (ICGIP 2014) (Vol. 9443, p. 94431R). International Society for Optics and Photonics.

18. Gamahara, M., \& Hattori, Y. (2017). Searching for turbulence models by artificial neural network. Physical Review Fluids, 2(5), 054604.

19. Kutz, J. N. (2017). Deep learning in fluid dynamics. Journal of Fluid Mechanics, 814, 1-4.

20. Lee, Y., Yang, H., \& Yin, Z. (2017). PIV-DCNN: cascaded deep convolutional neural networks for particle image velocimetry. Experiments in Fluids, 58(12), 171. 
21. Ling, J., Kurzawski, A., \& Templeton, J. (2016). Reynolds averaged turbulence modelling using deep neural networks with embedded invariance. Journal of Fluid Mechanics, 807, 155-166.

22. Newby, J. M., Schaefer, A. M., Lee, P. T., Forest, M. G., \& Lai, S. K. (2018). Convolutional neural networks automate detection for tracking of submicron-scale particles in 2D and 3D. Proceedings of the National Academy of Sciences, 115(36), 9026-9031.

23. Rabault, J., Kolaas, J., \& Jensen, A. (2017). Performing particle image velocimetry using artificial neural networks: a proof-of-concept. Measurement Science and Technology, 28(12), 125301.

24. Raissi, M., Wang, Z., Triantafyllou, M. S., \& Karniadakis, G. E. (2019). Deep learning of vortex-induced vibrations. Journal of Fluid Mechanics, 861, 119-137.

25. Strofer, C. M., Wu, J. L., Xiao, H., \& Paterson, E. (2019). Data-driven, physics-based feature extraction from fluid flow fields using convolutional neural networks. Communications in Computational Physics, 25(3), 625-650.

26. Yan, Y., Safdari, A., \& Kim, K. C. (2020). Visualization of nanofluid flow field by adaptive-networkbased fuzzy inference system (ANFIS) with cubic interpolation particle approach. Journal of Visualization, 1-9.

27. Xu, P., Babanezhad, M., Yarmand, H., \& Marjani, A. (2020). Flow visualization and analysis of thermal distribution for the nanofluid by the integration of fuzzy c-means clustering ANFIS structure and CFD methods. Journal of Visualization, 23(1), 97-110.

28. Jux, C. (2017). Robotic Volumetric Particle Tracking Velocimetry by Coaxial Imaging and Illumination.

29. Takagi, T., \& Sugeno, M. (1985). Fuzzy identification of systems and its applications to modeling and control. IEEE transactions on systems, man, and cybernetics, (1), 116-132.

\section{Figures}



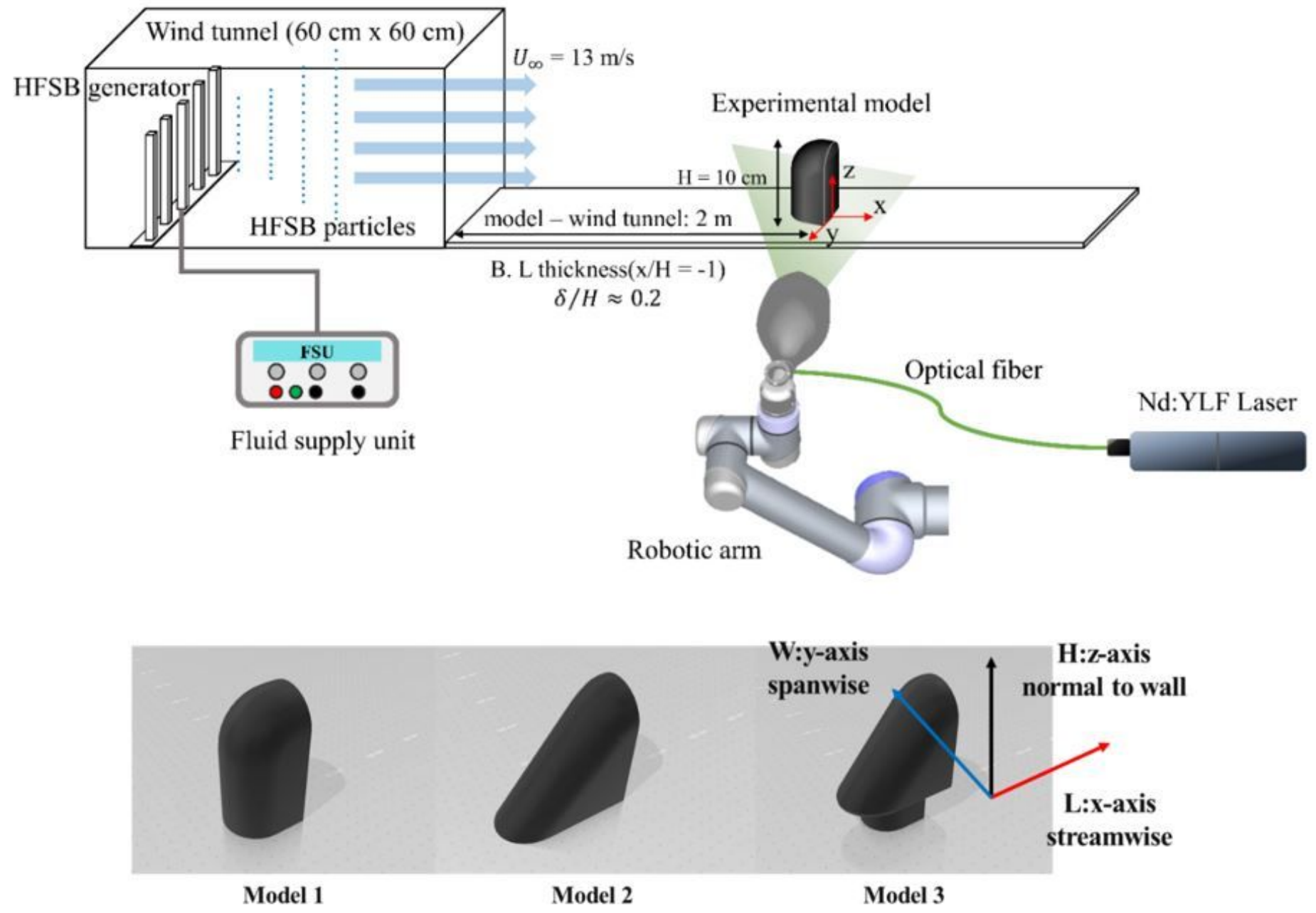

\section{Figure 1}

Experimental setup for time-resolved Lagrangian particle tracking velocimetry. 


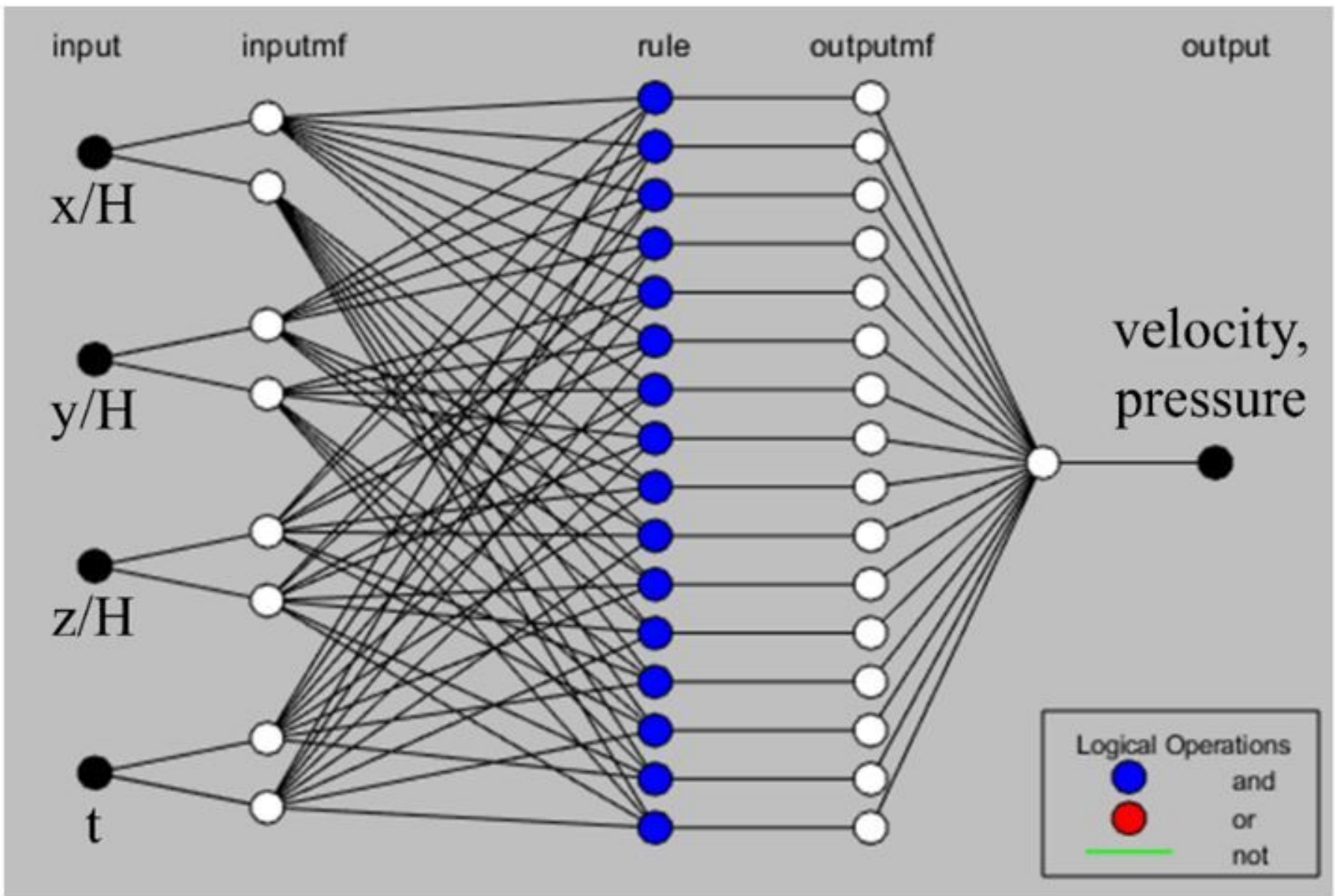

Figure 2

ANFIS structure with a four-input first-order Sugeno fuzzy model. 


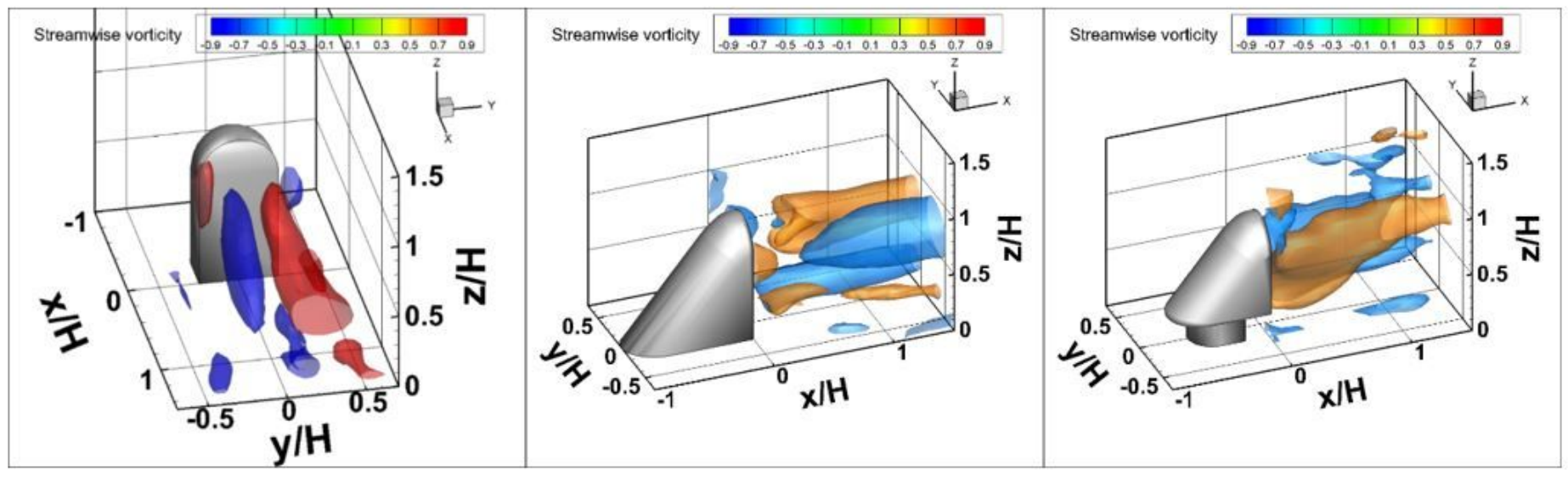

(a) Raw data on model 1(left); model 2(middle); model 3(right)

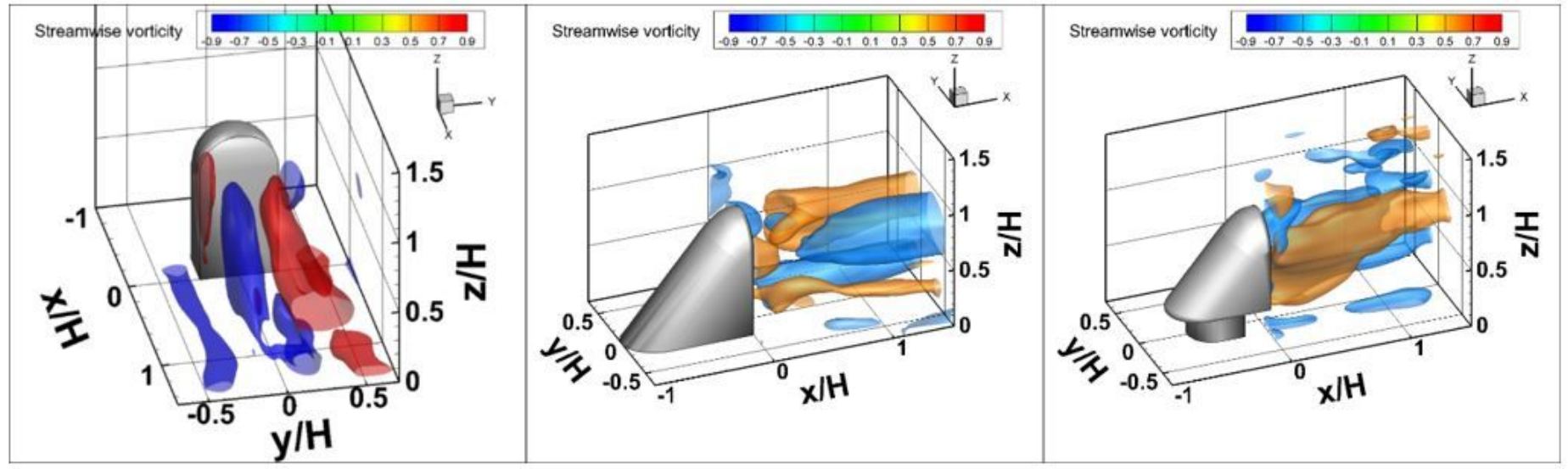

(b) Step size $x 8$ by ANFIS on model 1 (left); model 2 (middle); model 3 (right)

Figure 3

Comparison of the ensemble-averaged 3D streamwise vortex structures between the raw and assimilated data. 


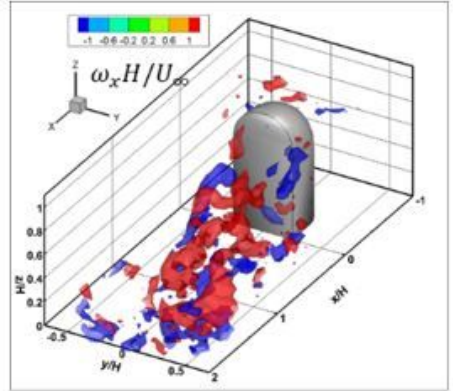

(a) Raw data ( $\left.t_{1}\right)$

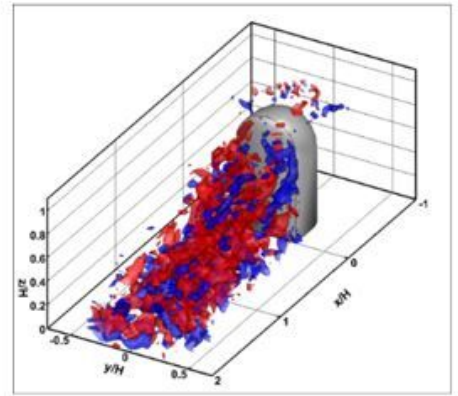

(c) ANFIS ( $t_{1}$, step size $\left.x 4\right)$

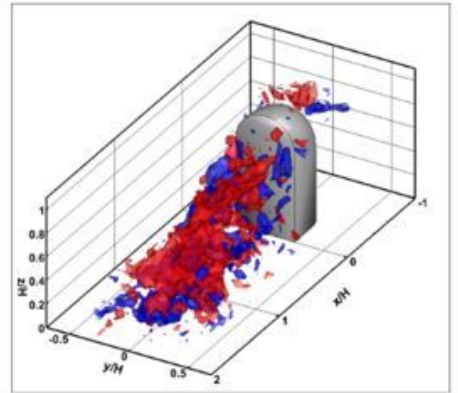

(d) ANFIS ( $t_{1}+d t$, step size $\left.x 4\right)$

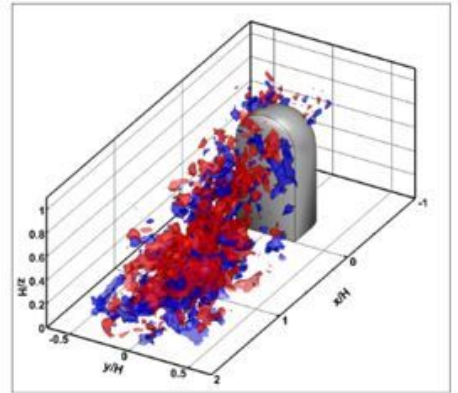

(e) ANFIS ( $t_{1}+2 d t$, step size $x$ 4)

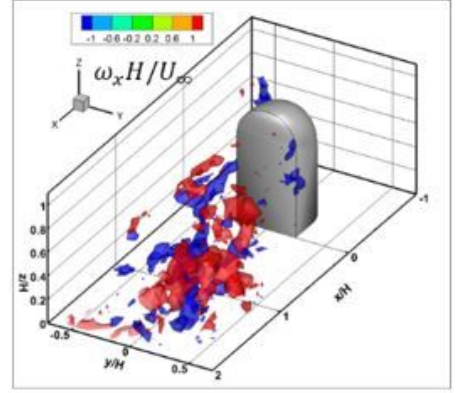

(b) Raw data $\left(t_{2}\right)$

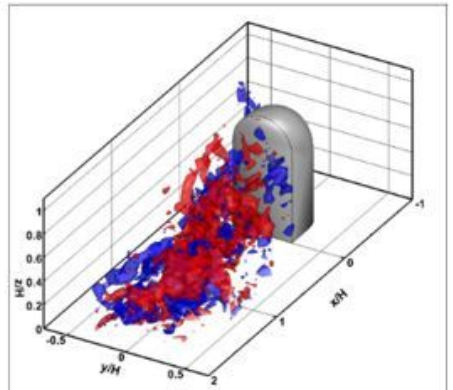

(f) ANFIS ( $t_{2}$, step size $\left.x 4\right)$

\section{Figure 4}

Comparison of the instantaneous 3D streamwise vortex structures between the raw and assimilated data. 


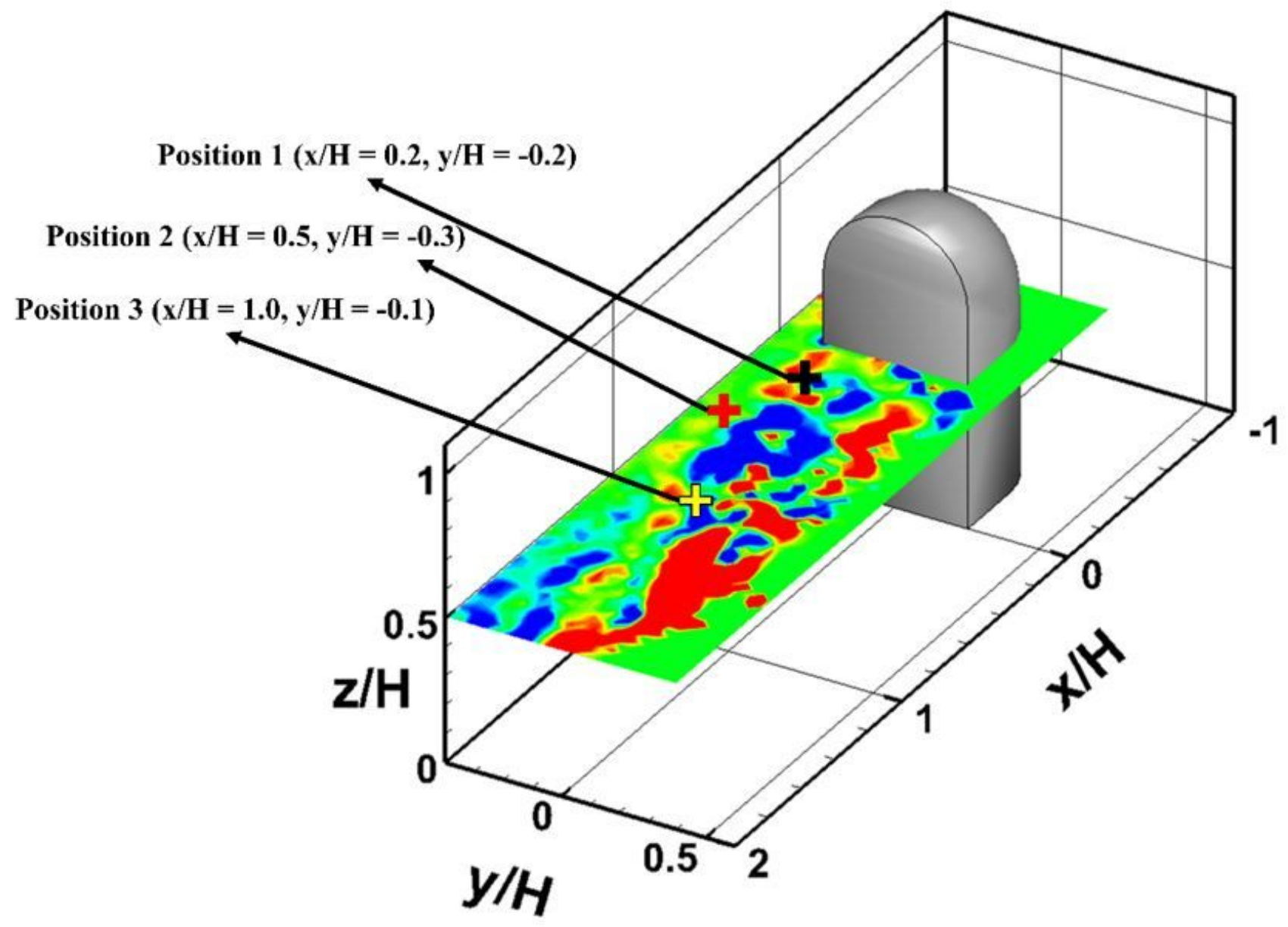

Figure 5

Measurement positions at $\mathrm{z} / \mathrm{H}=0.5$. 
(a)

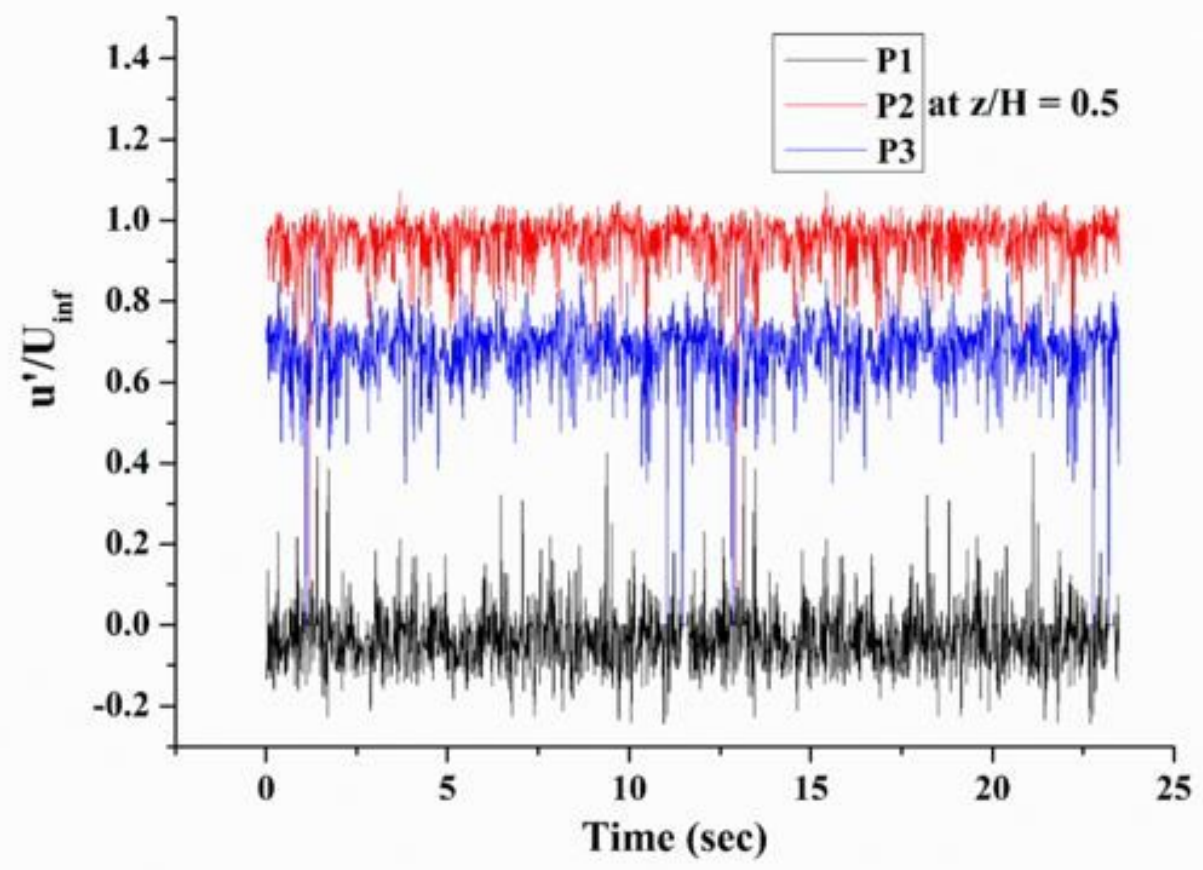

(b)

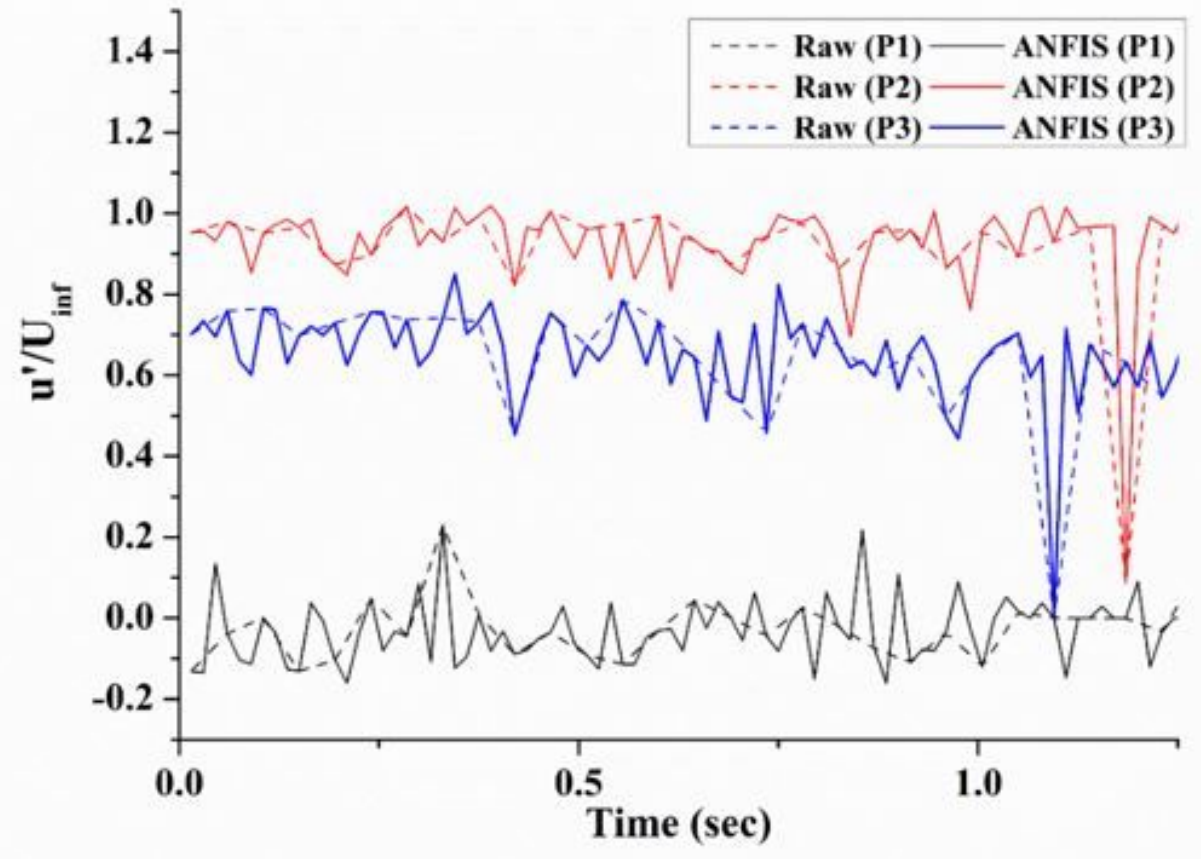

Figure 6

Comparison of the instantaneous streamwise velocity fluctuations between the raw and assimilated data taken at P1, P2, and P3 in the horizontal plane at $\mathrm{z} / \mathrm{H}=0.5$ for model 1. 


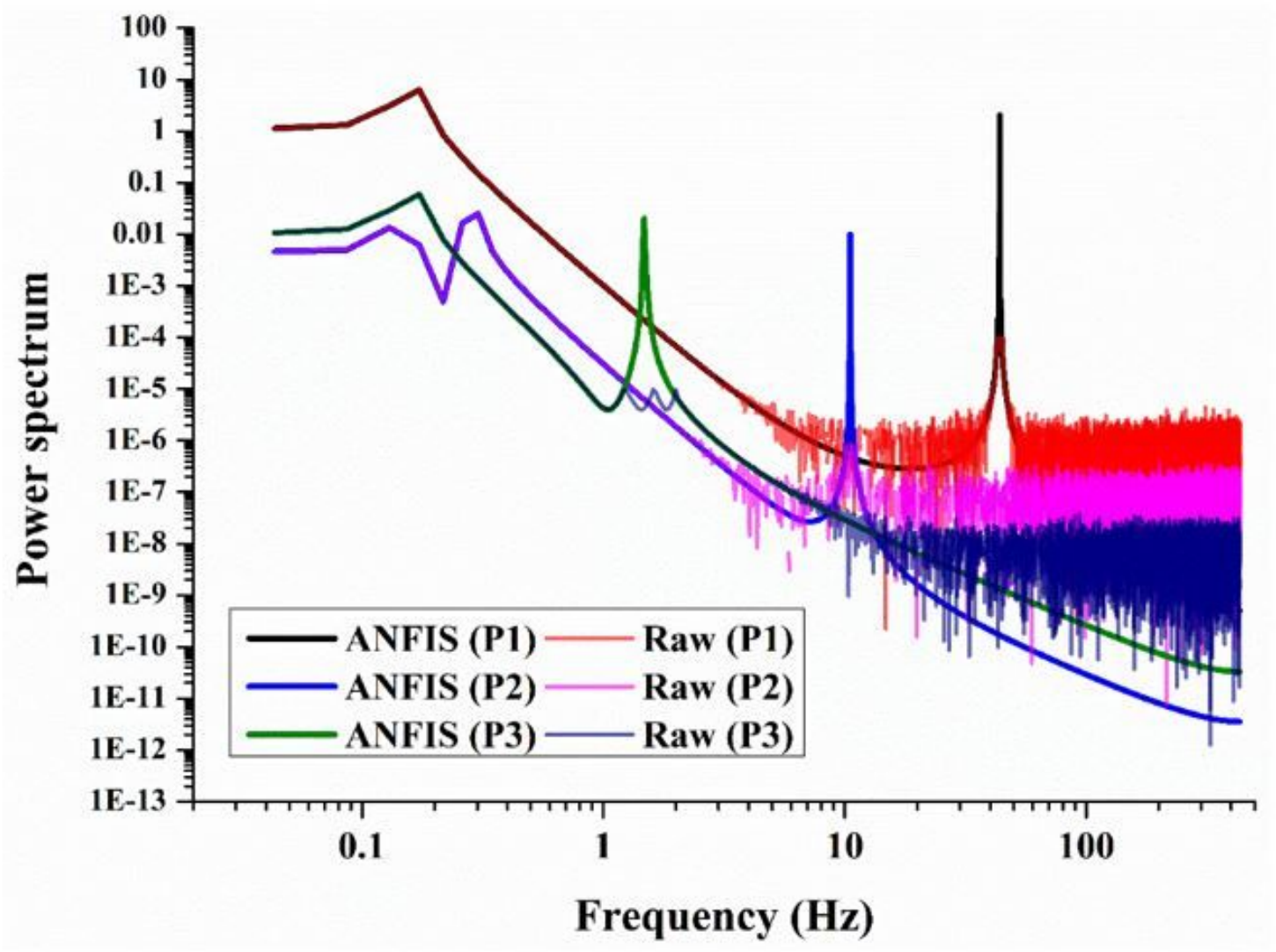

Figure 7

Comparison of the power spectrum from the instantaneous streamwise velocity fluctuation between the raw and assimilated data taken at $\mathrm{P} 1, \mathrm{P} 2$, and $\mathrm{P} 3$ in the horizontal plane at $\mathrm{z} / \mathrm{H}=0.5$ for model 1 . 


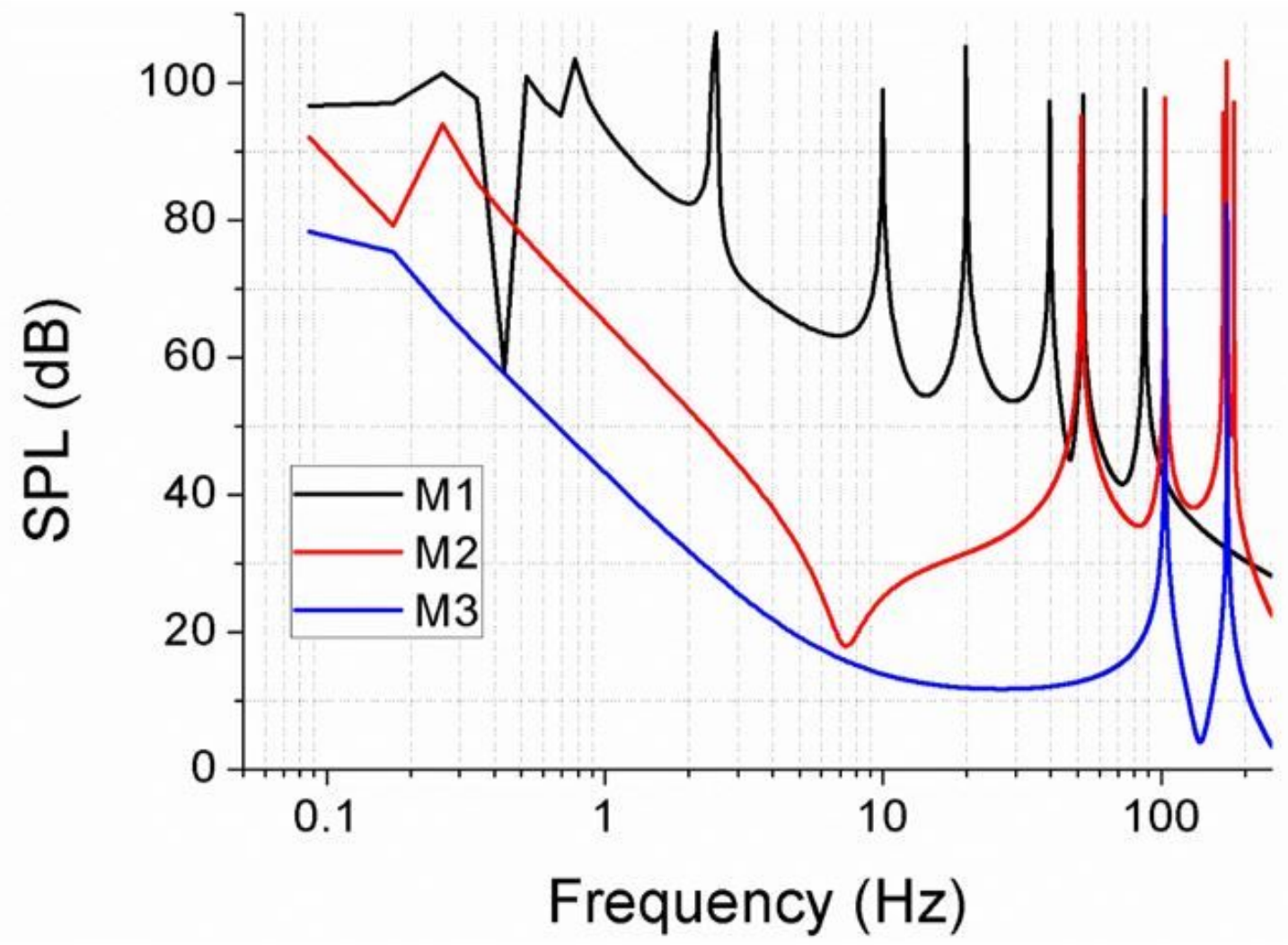

Figure 8

Comparison of assimilated sound pressure level at P1 for three different side mirror models. 